\title{
Myocardial ASL perfusion reserve test detects ischemic segments in initial cohort of 10 patients with angiographic CAD
}

\author{
Zungho Zun ${ }^{1 *}$, Terrence Jao ${ }^{1}$, Ning Smith², Padmini Varadarajan ${ }^{3}$, Ramdas G Pai ${ }^{3}$, Eric C Wong ${ }^{4}$, Krishna S Nayak \\ From 2011 SCMR/Euro CMR Joint Scientific Sessions \\ Nice, France. 3-6 February 2011
}

\section{Objective}

This study sought to demonstrate the potential for myocardial arterial spin labeling (ASL) to identify the ischemic myocardial segments due to stenosis in coronary arteries as detected by X-ray angiography.

\section{Background}

Myocardial ASL is a technique for the assessment of myocardial blood flow (MBF) without contrast agents. It can be safely applied to patients with end-stage renal disease who are not candidates for first-pass imaging with contrast agents. Myocardial ASL perfusion imaging performed at rest and during adenosine stress provides perfusion reserve $\left(\mathrm{MBF}_{\text {stress }} / \mathrm{MBF}_{\text {rest }}\right)$, which is a common indicator for the severity of coronary artery disease. In healthy myocardium, perfusion reserve is known to be approximately four [1].

\section{Methods}

Twenty nine patients were recruited from those scheduled for routine cardiac MR (CMR) and X-ray angiography. Myocardial ASL measurements were obtained from a single mid short-axis slice at rest and during adenosine infusion (dosage: $0.14 \mathrm{mg} / \mathrm{kg} / \mathrm{min}$ ) on a GE Signa 3T scanner. The ASL sequence was composed of flow-sensitive alternating inversion recovery (FAIR) tagging and balanced steady-state free precession (SSFP) imaging [2]. Perfusion reserve maps were generated in a standard short-axis view illustration by convolution with a Gaussian filter and resampling onto a polar coordinate [3].

\section{Results}

Ten of the twenty-nine patients were found to have significant stenosis on X-ray angiography. Table 1 contains the most ischemic myocardial segments in these ten patients as identified by two cardiologists using either $\mathrm{X}$-ray angiogram or ASL perfusion reserve map independently. Based on McNemar's test with Bonferroni correction, there was no significant difference between $\mathrm{X}$-ray and ASL MRI in identifying ischemia in all six myocardial segments $(\mathrm{p}=1.0000,0.6170,0.4795,0.1336$, 0.4795 , and 0.4795). Figure 1 contains perfusion reserve maps acquired using myocardial ASL in these patients. The average standard deviation of physiological noise

Table 1 Most ischemic myocardial segments identified by $X$-ray angiograms and by ASL perfusion reserve maps

\begin{tabular}{|c|c|c|c|}
\hline \multirow[t]{2}{*}{ Pts \# } & \multicolumn{2}{|c|}{ X-ray angiography } & \multirow{2}{*}{$\begin{array}{l}\text { ASL MRI } \\
\text { Ischemic } \\
\text { myocardial } \\
\text { segments }\end{array}$} \\
\hline & $\begin{array}{l}\text { Worst lesion on } \\
\text { angiogram }\end{array}$ & $\begin{array}{l}\text { Ischemic } \\
\text { myocardial } \\
\text { segments }\end{array}$ & \\
\hline 1 & Proximal LAD 100\% & Anterior & Anterior \\
\hline 2 & RCA $100 \%$ & Inferior, inferolateral & Inferolateral \\
\hline 3 & LAD 90\% & Anterior & $\begin{array}{l}\text { Anterior, } \\
\text { anteroseptal }\end{array}$ \\
\hline 4 & LCS 90\% (PDA) & $\begin{array}{l}\text { Inferoseptal, } \\
\text { inferior, inferolateral }\end{array}$ & Inferoseptal, inferior \\
\hline 5 & RCA (100\%) & Inferoseptal, inferior & $\begin{array}{l}\text { Anteroseptal, } \\
\text { inferoseptal, inferior }\end{array}$ \\
\hline 6 & RCA (100\%) & Inferoseptal, inferior & Inferior \\
\hline 7 & Distal RCA 80\% & Inferior & Anterolateral \\
\hline 8 & $\begin{array}{l}\text { Stent to } L A D \text { and } \\
\text { RCA - now open }\end{array}$ & $\begin{array}{l}\text { Anteroseptal, } \\
\text { inferoseptal }\end{array}$ & Anterior \\
\hline 9 & LCX $100 \%$, RCA $100 \%$ & $\begin{array}{l}\text { Inferior, } \\
\text { inferolateral, } \\
\text { anterolateral }\end{array}$ & Inferolateral \\
\hline 10 & RCA $100 \%$ & Inferior, inferolateral & Anteroseptal \\
\hline
\end{tabular}



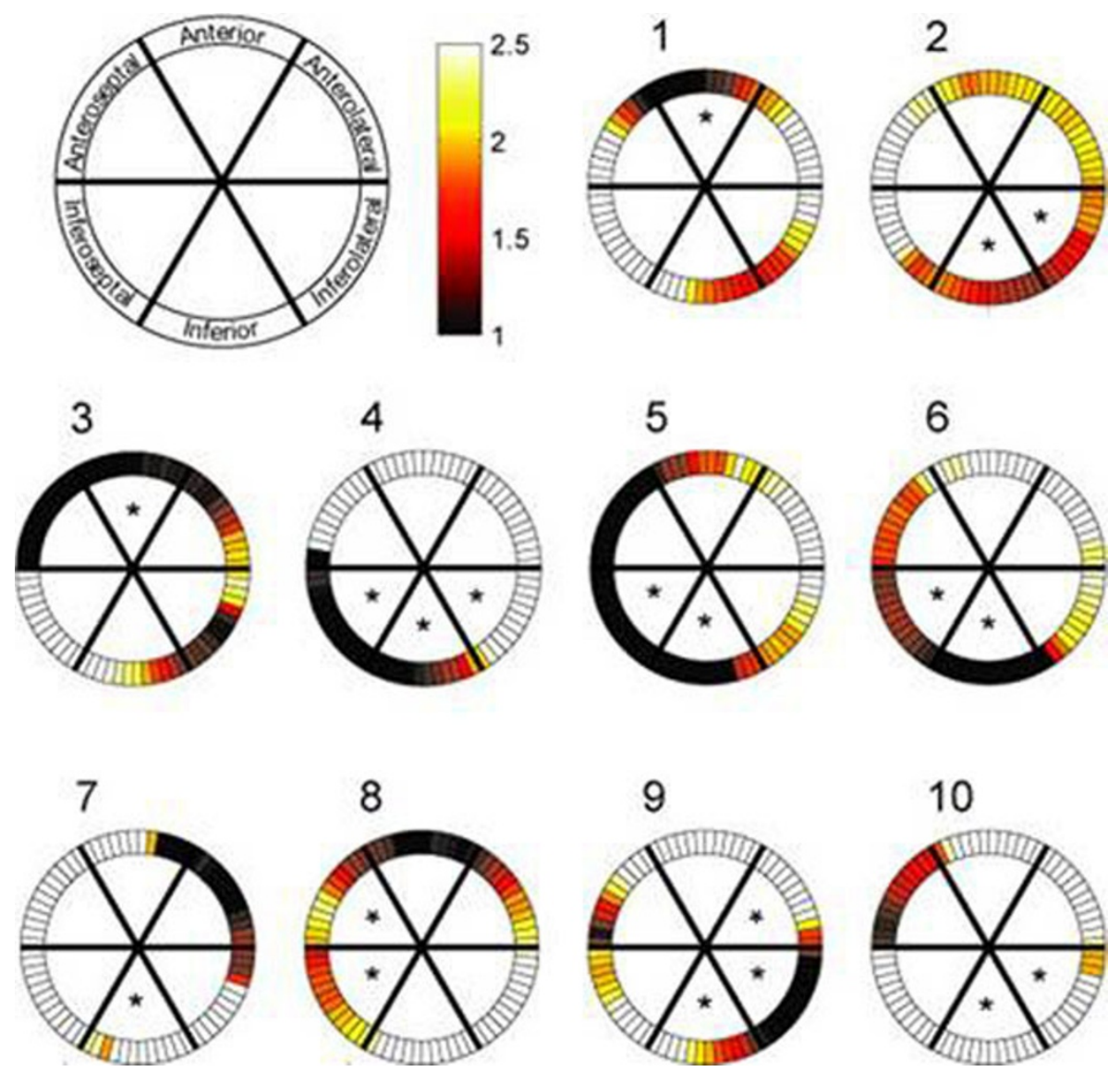

Figure 1 Perfusion reserve maps acquired using myocardial ASL in patients 1-10. Asterisks denote the most ischemic segments identified based on X-ray angiography.

was $0.22 \mathrm{ml} / \mathrm{g} / \mathrm{min}$ at rest and $0.42 \mathrm{ml} / \mathrm{g} / \mathrm{min}$ during stress [2].

\section{Conclusion}

There was visual agreement (except patients 7, 8, and 10) and no statistically significant difference between ischemic myocardial segments identified by ASL perfusion reserve maps and by $\mathrm{X}$-ray angiograms. This suggests that myocardial ASL with vasodilation may have a potential to identify ischemic myocardial segments in patients with stenosis.

\section{Author details}

${ }^{1}$ University of Southern California, Los Angeles, CA, USA. ${ }^{2}$ Kaiser Permanente Southern California, Pasadena, CA, USA. ${ }^{3}$ Loma Linda University Medical Center, Loma Linda, CA, USA. ${ }^{4}$ University of California, San Diego, La Jolla, CA, USA.

\section{Published: 2 February 2011}

\section{References}

1. Kaufmann, et al: Am J Physiol Heart Circ Physiol 2007.

2. Zun, et al: MRM 2009.

3. Jao, et al:, US patent (pending).
doi:10.1186/1532-429X-13-S1-P110

Cite this article as: Zun et al:: Myocardial ASL perfusion reserve test detects ischemic segments in initial cohort of 10 patients with angiographic CAD. Journal of Cardiovascular Magnetic Resonance 201113 (Suppl 1):P110.
Submit your next manuscript to BioMed Central and take full advantage of:

- Convenient online submission

- Thorough peer review

- No space constraints or color figure charges

- Immediate publication on acceptance

- Inclusion in PubMed, CAS, Scopus and Google Scholar

- Research which is freely available for redistribution 